\title{
ANALYTIC GENERATORS AND THE KMS CONDITION
}

\author{
JEAN DE CANNIËRE
}

\begin{abstract}
The KMS condition at arbitrary $\beta$ for a state $\omega$ of a $C^{*}$-dynamical system $(A, \mathbf{R}, \alpha)$ is stated in terms of the analytic continuation of the correlation functions $t \mapsto \omega\left(x^{*} \alpha_{t}(x)\right)(x \in A)$ in $-i$ (or $\left.+i\right)$. The precise formulation involves the analytic generator of $\alpha$ (or its inverse).
\end{abstract}

1. Introduction. Let $\alpha: t \mapsto \alpha_{t}$ be a strongly continuous one-parameter group of *-automorphisms of a $C^{*}$-algebra $A, \omega$ a state of $A, \beta$ a nonzero positive real number. We say that $\omega$ satisfies the KMS condition at $\beta$ (or is $\beta$-KMS) with respect to $\alpha[6 ; 2$, Chapter 5] if for every pair $x, y$ of elements of $A$ there exists a complex function $F$, defined, bounded and continuous on the strip $D_{\beta}=\{z \mid 0 \leqslant \operatorname{Im} z \leqslant \beta\}$, analytic in its interior, and with boundary values

$$
F(t)=\omega\left(y \alpha_{t}(x)\right), \quad F(t+i \beta)=\omega\left(\alpha_{t}(x) y\right) \quad(t \in \mathbf{R}) .
$$

On the other hand, denote by $\mathscr{D}(B)$ the space of all $x$ in $A$ for which there exists a (unique, and necessarily bounded) weakly continuous function $f_{x}$, defined on $D_{-1}=$ $\{z \mid-1 \leqslant \operatorname{Im} z \leqslant 0\}$ and with values in $A$, analytic in the interior of $D_{-1}$ and such that $f_{x}(t)=\alpha_{t}(x)$ for all real $t$. Define the analytic generator $B$ of $\alpha[3]$ by

$$
B x=f_{x}(-i) \quad(x \in \mathscr{Q}(B)) .
$$

Then $B$, with dense domain $\mathscr{D}(B)$, is a closed operator [3, Theorem 2.4] and completely determines $\alpha$ [3, Theorem 4.2].

These notions can be connected as follows. Suppose that $\omega$ is $\beta$-KMS, let $x$ be in $\mathscr{D}(B)$ and put $y=x^{*}$ in (1). For all $z$ with $-1 \leqslant \operatorname{Im} z \leqslant \beta$, define $G(z)=\omega\left(x^{*} f_{x}(z)\right)$ if $z \in D_{-1}$, and $G(z)=F(z)$ if $z \in D_{\beta}$. Then $G$ is bounded and continuous on the strip $D_{-1} \cup D_{\beta}$ and analytic in its interior. Using the $\alpha$-invariance of $\omega$ (which is a consequence of the KMS condition [2, Proposition 5.3.3]), one can check that

$$
G(t-i)=\omega\left(\left(f_{x}(-i / 2)\right) * \alpha_{t}\left(f_{x}(-i / 2)\right)\right)
$$

for all real $t$, whereas the KMS condition itself implies

$$
G(t+i \beta)=\omega\left(\alpha_{t}(x) x^{*}\right) .
$$

The two functions $t \mapsto G(t-i)$ and $t \mapsto G(t+i \beta)$ are then easily seen to be positive definite on the real line, again by the $\alpha$-invariance of $\omega$. Hence they attain

\footnotetext{
Received by the editors April 24, 1982.

1980 Mathematics Subject Classification. Primary 46L55; Secondary 30C80, 81A15.

'On leave from Nationaal Fonds voor Wetenschappelijk Onderzoek and Katholieke Universiteit Leuven, Belgium.
} 
their maximal absolute value at 0 . An application of the "three lines lemma" [9, Theorem 12.8] yields

$$
|G(0)|^{\beta+1} \leqslant G(-i)^{\beta} G(i \beta)
$$

or, in view of (1) and (2),

$$
\omega\left(x^{*} x\right)^{\beta+1} \leqslant \omega\left(x^{*} B x\right)^{\beta} \omega\left(x x^{*}\right) .
$$

It is the purpose of this note to show that the validity of (3) for all $x$ in $\mathscr{D}(B)$ is also sufficient to ensure that an $\alpha$-invariant state $\omega$ be $\beta$-KMS ( $\$ 2)$. The method developed below can actually be used to derive a variety of reformulations of the KMS condition (\$3). Some of these are already known in quantum statistical mechanics [2, Chapter 5], others might be of interest there as well.

2. The main result. Let us agree upon the definition $\hat{f}(\lambda)=\int e^{i \lambda t} f(t) d t(\lambda \in \mathbf{R})$ for the Fourier transform $\hat{f}$ of $f$ in $L^{1}(\mathbf{R})$. Given any open interval $(\xi, \eta)(\xi, \eta \in \mathbf{R} \cup$ $\{ \pm \infty\}$ ), define the spectral subspace $R(\xi, \eta)$ of $A$ (associated with $\alpha$ ) to be the closed linear span in $A$ of all elements of the form $\int f(t) \alpha_{t}(x) d t$, where $x \in A, f \in L^{1}(\mathbf{R})$ and the support of $\hat{f}$ is contained in $(\xi, \eta)$. The following form of the KMS condition has proved useful in [4].

Lemma 1 [4, Theorem 1.1]. Let $\omega$ be a state of $A$, and let $0<\beta<+\infty$. Then the following are equivalent:

(i) $\omega$ is $\beta$-KMS with respect to $\alpha$;

(ii) $\omega$ is $\alpha$-invariant, and $\omega\left(x^{*} x\right) \leqslant e^{\beta \xi} \omega\left(x x^{*}\right)$ whenever $x \in R(-\infty, \xi), \xi \in \mathbf{R}$.

If $\omega$ is an $\alpha$-invariant state of $A$, and $x \in A$, then by Bochner's theorem there exist unique bounded positive Radon measures $\mu_{x}$ and $\nu_{x}$ on $\mathbf{R}$ satisfying

$$
\omega\left(x^{*} \alpha_{t}(x)\right)=\int e^{i \lambda t} d \mu_{x}(\lambda) \text { and } \omega\left(\alpha_{t}(x) x^{*}\right)=\int e^{i \lambda t} d \nu_{x}(\lambda)
$$

for all $t$ in $\mathbf{R}$. These measures are instrumental in formulating the following theorem.

THEOREM 1. Let $\omega$ be an $\alpha$-invariant state of $A$. Suppose that there exist a strictly increasing function $\phi:(0,+\infty) \rightarrow \mathbf{R}$ and a subset $A_{0}$ of $A$ such that the following conditions hold:

(i) For all $\xi$ in $\mathbf{R}$, the spectral subspace $R(-\infty, \xi)$ is the norm-closure of $R(-\infty, \xi) \cap$ $A_{0}$.

(ii) For all $x$ in $A_{0}$, the function $\lambda \mapsto \phi\left(e^{-\beta \lambda}\right) \vee 0$ on $\mathbf{R}$ is $\mu_{x}$-integrable.

(iii) If $x \in A_{0}$ and $\omega\left(x^{*} x\right) \neq 0$, then

$$
\omega\left(x^{*} x\right) \phi\left(\omega\left(x x^{*}\right) \omega\left(x^{*} x\right)^{-1}\right) \geqslant \int \phi\left(e^{-\beta \lambda}\right) d \mu(\lambda),
$$

where we define $\phi(0)=\lim _{\lambda \geqq 0} \phi(\lambda) \in \mathbf{R} \cup\{-\infty\}$.

Then $\omega$ is $\beta-K M S$ with respect to $\alpha$. If instead of (i) we have

(i)' if $\xi \in \mathbf{R}$, then $\overline{R(\xi,+\infty) \cap A_{0}}=R(\xi,+\infty)$,

and we replace $\vee$ with $\wedge$ in (ii) and $\geqslant$ with $\leqslant$ in (iii), we obtain the same conclusion.

Proof. Let $\omega$ be an $\alpha$-invariant state, and let $A_{0}$ and $\phi$ satisfy the first set of assumptions of the theorem. If $x \in R(-\infty, \xi)$, it is easy to see that supp $\mu_{x} \subset(-\infty, \xi]$ 
[4, Lemma 1.6]. Thus if $x \in R(-\infty, \xi) \cap A_{0}$, then one has

$$
\int \phi\left(e^{-\beta \lambda}\right) d \mu_{x}(\lambda) \geqslant \phi\left(e^{-\beta \xi}\right) \int d \mu_{x}(\lambda)=\phi\left(e^{-\beta \xi}\right) \omega\left(x^{*} x\right),
$$

because $\phi$ is increasing. If, moreover, $\omega\left(x^{*} x\right) \neq 0$, then (iii) implies $\phi\left(\omega\left(x x^{*}\right) \omega\left(x^{*} x\right)^{-1}\right) \geqslant \phi\left(e^{-\beta \xi}\right)$, and hence

$$
\omega\left(x x^{*}\right) \geqslant e^{-\beta \xi} \omega\left(x^{*} x\right),
$$

where we used the fact that $\phi$ is strictly increasing. But (5) holds trivially if $\omega\left(x^{*} x\right)=0$ as well, and furthermore by (i) it is valid for all $x$ in $R(-\infty, \xi)$. Since $\xi$ was arbitrary, the state $\omega$ is $\beta$-KMS by Lemma 1 . The second part of the theorem can be proved in a similar way, once it is observed that $R(\xi,+\infty)^{*}=-R(-\infty,-\xi)$.

In the application of the above theorem to the problem stated in the Introduction, the role of $A_{0}$ will be played by the space of all $\alpha$-analytic elements of $A$ (we say that an element $x$ of $A$ is $\alpha$-analytic if the function $t \mapsto \alpha_{t}(x)$ on $\mathbf{R}$ extends to an entire analytic function on $\mathbf{C}$ ). With this definition, clearly $A_{0} \subset \mathcal{D}(B)$, and, moreover, $A_{0}$ satisfies both (i) and (i)' of Theorem 1. To see this, define Gaussian kernels $g_{n}$ by

$$
g_{n}(z)=(n / \pi)^{1 / 2} \exp \left(-n z^{2}\right) \quad\left(n \in \mathbf{N}_{0}, z \in \mathbf{C}\right),
$$

which are entire analytic. Then for $x$ in any $\mathbf{R}(\xi, \eta)$, the elements $\int g_{n}(t) \alpha_{t}(x) d t$ of $A$ are $\alpha$-analytic, they belong to $R(\xi, \eta)$, arid they tend in norm to $x$ as $n \rightarrow \infty[2$, Proposition 2.5.22]. Finally we also need the following simple lemma.

LEMMA 2. Let $f: D_{-1} \rightarrow \mathbf{C}$ be a bounded continuous function that is analytic in the interior of $D_{-1}$, and whose restriction to the real line is positive definite. If $\mu$ is the unique bounded positive Radon measure on $\mathbf{R}$ such that $\int e^{i \lambda t} d \mu(\lambda)=f(t)$ for all $t$ in $\mathbf{R}$, then the real function $\lambda \mapsto e^{\lambda}$ is $\mu$-integrable, and $\int e^{\lambda} d \mu(\lambda)=f(-i)$.

Proof. As $f$ is continuous and bounded, we have, with $g_{n}$ as in (6), that $f(-i)=\lim _{n \rightarrow \infty} \int f(t-i) g_{n}(t) d t$. Let $s$ denote a real number in $(-1,0)$. By the dominated convergence theorem,

$$
\int f(t-i) g_{n}(t) d t=\lim _{s \geqq-1} \int f(t+i s) g_{n}(t+i(s+1)) d t
$$

for all $n$ in $\mathbf{N}_{0}$. But by Cauchy's theorem the integrals in the right-hand side are independent of $s$. In particular,

$$
\begin{aligned}
\int f(t-i) g_{n}(t) d t & =\lim _{s \leq 0} \int f(t+i s) g_{n}(t+i(s+1)) d t \\
& =\int f(t) g_{n}(t+i) d t
\end{aligned}
$$

again by the dominated convergence theorem. Furthermore,

$$
\int f(t) g_{n}(t+i) d t=\int \exp \left(-\lambda^{2} / 4 n+\lambda\right) d \mu(\lambda),
$$


as one easily computes. Hence by the monotone convergence theorem the function $\lambda \mapsto e^{\lambda}$ is $\mu$-integrable and

$$
\int e^{\lambda} d \mu(\lambda)=\lim _{n \rightarrow \infty} \int \exp \left(-\lambda^{2} / 4 n+\lambda\right) d \mu(\lambda)=f(-i)
$$

THEOREM 2. Let $\omega$ be an $\alpha$-invariant state of $A$, and $\beta$ a nonzero positive number. If $\omega$ is $\beta$-KMS with respect to $\alpha$, then the inequality

$$
\omega\left(x^{*} x\right)^{\beta+1} \leqslant \omega\left(x^{*} B x\right)^{\beta} \omega\left(x x^{*}\right)
$$

holds for all $x$ in the domain of the analytic generator $B$ of $\alpha$. Conversely, if (7) is valid for every $\alpha$-analytic $x$ in $A$, then $\omega$ is $\beta-K M S$ with respect to $\alpha$.

Proof. If $x \in \mathcal{Q D}(B)$, we can apply Lemma 2 with $f(z)=\omega\left(x^{*} f_{x}(z)\right)$ and $\mu=\mu_{x}$ to obtain that the function $\lambda \mapsto e^{\lambda}$ is $\mu_{x}$-integrable, and $\int e^{\lambda} d \mu_{x}(\lambda)=\omega\left(x^{*} B x\right)$. Suppose $x$ is $\alpha$-analytic and (7) holds. Define a strictly increasing function $\phi$ : $(0,+\infty) \rightarrow \mathbf{R}$ by $\phi(t)=-t^{-1 / \beta}$. If $\omega\left(x^{*} x\right) \neq 0$, then inequality (7) implies that $\omega\left(x x^{*}\right) \neq 0$, hence it can be rewritten as

$$
\omega\left(x^{*} x\right)^{1+1 / \beta} \omega\left(x x^{*}\right)^{-1 / \beta} \leqslant \omega\left(x^{*} B x\right),
$$

or

$$
\omega\left(x^{*} x\right) \phi\left(\omega\left(x x^{*}\right) \omega\left(x^{*} x\right)^{-1}\right) \geqslant \int \phi\left(e^{-\beta \lambda}\right) d \mu_{x}(\lambda) .
$$

By Theorem 1 (with the space of $\alpha$-analytic elements as $A_{0}$ ) $\omega$ is $\beta$-KMS with respect to $\alpha$. This proves the sufficiency of the condition whose necessity was established in the Introduction.

3. Additional remarks. (a) It is worthwhile pointing out that the inequalities appearing in the statement of Theorem 1 actually follow from the KMS condition if $\phi$ is supposed to be convex or concave. Specifically, if $\omega$ is $\beta$-KMS with respect to $\alpha$, and if $\phi:(0,+\infty) \rightarrow \mathbf{R}$ is concave (not necessarily increasing), then for all $x$ in $A$ with $\omega\left(x^{*} x\right) \neq 0$ one has

$$
\omega\left(x^{*} x\right) \phi\left(\omega\left(x x^{*}\right) \omega\left(x^{*} x\right)^{-1}\right) \geqslant \int \phi\left(e^{-\beta \lambda}\right) d \mu_{x}(\lambda) .
$$

If $\phi$ is convex the opposite inequality obtains. This follows immediately from Jensen's inequality [9, Theorem 3.3], and from the well-known fact [2, Proposition 5.3.14] that the KMS condition at $\beta$ implies that $\mu_{x}$ and $\nu_{x}$ are equivalent measures for all $x$ in $A$, with Radon-Nikodým derivative given by $\left(d \mu_{x} / d \nu_{x}\right)(\lambda)=e^{\beta \lambda}$ for $\nu_{x}$-almost all $\lambda$ in $\mathbf{R}$.

Together with Theorem 1 , then, this observation yields a condition equivalent with the KMS condition for every strictly increasing concave (or convex) function $\phi$. The choices $\phi(t)=\log t$ and $\phi(t)=(t-1) / \log t$ lead to "correlation inequalities" that have appeared before in the physics literature (see [10, Theorem 3; 1, Theorem 1] for the first case, $[8, \S 2 ; 7$, Theorem III.1; 5, Theorem II.4] for the second case, and [2, §5.3.1] for a general survey of known results of this type). In view of these and related papers, it is actually clear that the method we suggest (i.e. the use of Fourier 
analysis and convexity) has essentially been known for some time, although not in any systematic form.

(b) Let us give another illustration of this general method. The analytic generator $B$ of $\alpha$ is invertible, and the domain $\mathscr{D}\left(B^{-1}\right)$ of its inverse $B^{-1}$ clearly consists of those $x$ in $A$ for which there exists a weakly continuous $A$-valued function $g_{x}$ on $D_{1}$ that is analytic in the interior of $D_{1}$ and satisfies $g_{x}(t)=\alpha_{t}(x)$ for all real $t$; moreover $B^{-1} x=g_{x}(i)$ [3, Theorem 2.4]. The relationship between $B^{-1}$ and the KMS condition is as follows:

(i) If $\omega$ is $\beta$-KMS with respect to $\alpha$ and $0<\beta \leqslant 1$, then

$$
\omega\left(x x^{*}\right) \leqslant \omega\left(x^{*} x\right)^{1-\beta} \omega\left(x^{*} B^{-1} x\right)^{\beta}
$$

for all $x$ in $92\left(B^{-1}\right)$. Conversely, if (8) holds for all $\alpha$-analytic $x$, then $\omega$ is $\beta$-KMS.

(ii) If $\omega$ is $\beta$-KMS with respect to $\alpha$ and $1 \leqslant \beta<+\infty$, then

$$
\omega\left(x^{*} B^{-1} x\right)^{\beta} \leqslant \omega\left(x^{*} x\right)^{\beta-1} \omega\left(x x^{*}\right)
$$

for all $x \in \ln \left(B^{-1}\right)$, which is exactly the opposite of (8). Again the converse is true as well.

The distinction between $\beta \leqslant 1$ and $\beta \geqslant 1$ can be traced back to the fact that the function $t \mapsto t^{1 / \beta}$ is convex on $(0,+\infty)$ in the first case, and concave in the second. Setting in particular $\beta=1$, we observe the somewhat surprising phenomenon that the statement

$$
\omega\left(x^{*} B^{-1} x\right) \geqslant \omega\left(x x^{*}\right) \text { for all } x \text { in } \circ D\left(B^{-1}\right)
$$

is equivalent with

$$
\left.\omega\left(x^{*} B^{-1} x\right) \leqslant \omega\left(x x^{*}\right) \text { for all } x \text { in } \mathcal{Q}\right)\left(B^{-1}\right),
$$

since both express the KMS condition at 1 .

(c) Although inequality (3) is not linear in $\omega$, every KMS state $\omega$ (at arbitrary $\beta$ ) can be shown to satisfy the linear inequality

$$
\omega\left(x^{*} B x\right)-\omega\left((B x) x^{*}\right) \geqslant \omega\left(x^{*} x\right)-\omega\left(x x^{*}\right)
$$

for all $x$ in $Q(B)$. This does not seem to follow immediately from (3). Rather one argues, using the idea of the proof of [4, Theorem 3.3], that (9) holds for all $x$ in $\mathscr{D}(B)$ if (and only if) $\omega\left(x^{*} x\right) \leqslant \omega\left(x x^{*}\right)$ for all $x$ in $R(-\infty, 0)$ (this latter condition is called "spectral passivity" in [4]). Then one uses Lemma 1.

\section{REFERENCES}

1. H. Araki, On KMS states of a $C^{*}$-dynamical system, Proc. Second Japan-U.S.A. Seminar on $C^{*}$-Algebras and Applications to Physics, Lecture Notes in Math., vol. 650, Springer-Verlag, Berlin and New York, 1978.

2. O. Bratteli and D. W. Robinson, Operator algebras and quantum statistical mechanics. II, Springer, New York, 1981.

3. I. Ciorănescu and L. Zsidó, Analytic generators for one-parameter groups, Tôhoku Math. J. 28 (1976), 327-362.

4. J. De Cannière, A spectral characterization of KMS states, Comm. Math. Phys. 84 (1982), 187-205.

5. M. Fannes and A. Verbeure, Correlation inequalities and equilibrium states. II, Comm. Math. Phys. 57 (1977), 165-171.

6. R. Haag, N. M. Hugenholtz and M. Winnink, On the equilibrium states in quantum statistical mechanics, Comm. Math. Phys. 5 (1967), 215-236. 
7. J. Naudts and A. Verbeure, Bounds on the admittance for KMS states, J. Math. Phys. 17 (1976), 419-423.

8. G. Roepstorff, Correlation inequalities in quantum statistical mechanics and their application in the Kondo problem, Comm. Math. Phys. 46 (1976), 253-262.

9. W. Rudin, Real and complex analysis, McGraw-Hill, New York, 1974.

10. G. L. Sewell, KMS condition and local thermodynamic stability of quantum lattice systems. II, Comm. Math. Phys. 55 (1977), 53-61.

Department of Mathematics, University of California, Berkeley, California 94720

Current address: Departement Wiskunde, Katholieke Universiteit Leuven, Celestijnenlaan 200 B, B-3030 Leuven, Belgium 